\title{
PROYECTO TRANSNACIONALIZADOR: TISA Y SU IMPACTO EN LOS PROCESOS HISTÓRICOS, POLÍTICOS, ECONÓMICOS, SOCIALES Y HUMANOS.
}

TRANSNATIONALIZING PROJECT: TISA AND ITS IMPACT ON HISTORICAL, POLITICAL, ECONOMIC, SOCIAL AND HUMAN PROCESSES.

Kayrha Sánchez, kayrha_sanchez@yahoo.es; Marlene De León, itzel0469@gmail.com Saul Cortez, saulcortez77@yahoo.com; Florencio muñoz, fr-munozb@outlook.es

Universidad de Panamá, Asociación de Profesores de la Universidad de Panamá, Panamá, Panamá.

\section{RESUMEN}

El TISA (Trade in Services Agreement) es un acuerdo comercial desconocido, del que nadie habla, negociado con gran hermetismo; entre sus promotores encontramos aquellos que abogan por la apertura del "mercado de servicios" a la lógica del capital internacional. No escapa la educación, la salud, el transporte, las telecomunicaciones, servicios financieros, el agua potable, la protección de los datos de la ciudadanía; en síntesis, la democracia y las políticas públicas. El Acuerdo sobre el Comercio de Servicios (ACS) dentro del Trade in Services Agreement (TISA) integrado, por 50 países: E.E.U.U, la U.E. América Latina, Asia y otros; sin que sus poblaciones ni sus parlamentos expresen su punto de vista.

Las fuertes presiones internacionales amenazan gravemente la existencia de los servicios públicos, esenciales y no esenciales y agudiza las tentativas de regulación del sector financiero, en detrimento de más del $70 \%$ de la población mundial

PALABRAS ClAVE: Políticas públicas, Acuerdo Internacionales, Doctrina del Shock, Comercio de Servicios.

\begin{abstract}
The TISA (Trade in Services Agreement) is an unknown commercial agreement, which nobody talks about, negotiated with great secrecy; Among its promoters we find those who advocate the opening of the "service market" to the logic of international capital. Education, health, transportation, telecommunications, financial services, drinking water, the protection of citizens' data do not escape; in short, democracy and public policies. The Agreement on Trade in Services (ACS) within the Trade in Services Agreement (TISA) made up of 50 countries: the U.S., the U.E. Latin America, Asia and others; without their populations or their parliaments expressing their point of view.

Strong international pressure seriously threatens the existence of public services, essential and non-essential, and exacerbates attempts to regulate the financial sector, to the detriment of more than $70 \%$ of the world's population.
\end{abstract}


KEYWORDS: Public policies, International Agreement, Shock Doctrine, Trade in Services.

\section{INTRODUCCIÓN}

Naomi Klein en 2011, acuñó como categoría la Doctrina del Shock para revelar el proceso de privatización de países que afrontan momentos convulsos, producto de desastres naturales, crisis sanitarias, económicas o políticas Este "shock", sustentado en el liberalismo económico, surgido en la séptima década del siglo pasado en la Universidad de Chicago, por Milton Friedman (Lange, 2010), consistente en la oportunidad que aprovechan gobernantes de las tragedias como mecanismo de especulación para lograr reformas políticamente imposibles en tiempos normales pero en situaciones sociales trágicas se establecen sin mayores resistencias

En términos generales, lo que se vive en Panamá es la aplicación sin anestesia de la doctrina del shock, toda vez que su programa de gobierno permite colegir que se valdrá del ambiente social político de pandemia para impulsar las recetas neoliberales a través de reformas de carácter impopulares.

El TISA (Trade in Services Agreement, por sus siglas en inglés) cuya intención es liberalizar el comercio de servicios a nivel internacional. enmarcado al nivel de la Asociación Transatlántica de Comercio e Inversión (TTIP, por sus siglas en inglés). Sus principales impulsores son los E.E.UU., la U.E y la Asociación Trans- Pacífica (TPP, por sus siglas en inglés) alianza que ejecuta Estados Unidos y la región asiática (Abreu, 2015).

El TISA es una estructura política económica que en conjunto abarca aproximadamente $70 \%$ del comercio mundial de servicios. La divulgación de informaciones, proveniente de WikiLeaks (Assange, 2011), facilita configurar una panorámica más precisa de sus verdaderos objetivos y potenciales impactos dirigidos hacia las telecomunicaciones, el sector financiero, el transporte aéreo y marítimo, las modalidades de comercio electrónico, los servicios postales, la distribución de mercancías, el movimiento de personas y los servicios profesionales; igualmente determinadas normativas de carácter interno de los Estados. (Espinosa, 2016).

El artículo que sometemos a la consideración, análisis, debate de los lectores tiene el propósito de intercambiar opiniones sobre el TISA, su estructura, sus lineamientos generales, el 
impacto e implicaciones para Panamá, país cuya economía gravita más del 70\% del sector servicio, y por ende a los profesionales dedicados a sus distintas especialidades (Valdés, 2017).

\section{El acuerdo de comercialización de servicios.}

El Acuerdo sobre Comercio de Servicios (ACS o TISA, por sus siglas en inglés) está concebido como un tratado internacional de promoción de la apertura comercial dedicado exclusivamente a los servicios: Desde el 2013 se lleva a cabo una serie de negociaciones, a espalda de los pueblos y de la OMC, descubierto por los WikiLeaks revelándose las negociaciones secretas que llevaban adelante más de medio centenar de países. encabezado por Estados Unidos y la Unión Europea (esta última agrupa 28 países).

\section{Antecedentes.}

El acuerdo sobre el Comercio de Servicios (TISA) es el producto al estancamiento en las negociaciones comerciales de la OMC, destinadas a ampliar el Acuerdo General sobre el Comercio de Servicios (AGCS), denominado "los verdaderos buenos amigos de los servicios", firmes defensores de la liberalización del comercio.

Entre los 50 gobiernos presentes en las mesas de negociaciones se encuentran: Australia, Canadá, Chile, Colombia, Corea, Costa Rica, Estados Unidos, Hong Kong, Islandia, Israel, Japón, Liechtenstein, Mauricio, México, Nueva Zelanda, Noruega, Pakistán, Panamá, Perú, Suiza, Taiwán, Turquía y la Unión Europea. Hasta noviembre de 2016 se realizaron 21 rondas de negociaciones.

\section{Estructura del Acuerdo.}

"Texto central", consta de cuatro partes, cuyo principio central es desregularizar los principales servicios e instituciones de los Estados firmantes: salud educación transporte recursos naturales entre otros

\section{Parte I. Disposiciones Generales}

Enfocada en las obligaciones básicas, similar a lo dispuesto en el Acuerdo General de Comercio de Servicios -AGCS- de la OMC con exigencias superiores en cuanto a cobertura y control sectorial, se refiere incluyendo compromisos infra y supra nacionales.

\section{Parte II. Forma de Listar los Compromisos}


Establece la nueva forma de listar los compromisos de apertura (Acceso a Mercados y Trato Nacional), mediante la creación de Listas "Hibridas" de Compromisos Específicos: listas positivas para Acceso a Mercados (solo se libera lo que se incluye) y listas negativas para Trato Nacional (se libera todo salvo lo que exceptúe de manera expresa).

\section{Parte III. Disciplinas Nuevas y Mejoradas}

Enfoca las “Disciplinas Nuevas y Mejoradas" (New and Enhanced Disciplines), resalta los intereses de la industria por acaparar los espacios nacionales acordes a las necesidades de la lógica trasnacional, de la cual surgieron 12 propuestas siendo las más sobresalientes: Regulación Doméstica; Transparencia; Movimiento de Personas (Modo 4); Tics (telecomunicaciones y comercio electrónico); Servicios Financieros; Transporte Marítimo; Servicios Profesionales; Correo Competitivo; Servicios Aéreos; Servicios Energéticos; Subsidios; y Transporte de Carga por Carretera.

\section{DESARROLLO}

\section{Cláusulas que se imponen con el acuerdo TISA}

Fundamentado en las fuentes consultadas que involucra el Informe Especial de la Internacional de Servicios Públicos (ISP) y el informe "Un Plan de Acción para la Privatización Global", publicado por el portal Global Justice Now y el portal http//www.wikileaks.org/tisa y sus vínculos desagregados, logramos obtener algunas de las cláusulas bajo las cuales se están negociando los acuerdo. Para este análisis seleccionamos nueve de ellas que procederemos a describir y analizar.

1 clausula Trinquete. Esta disposición bloquea automáticamente cualquier medida futura adoptada para liberalizar los servicios de un determinado país, pues establece que cualquier cambio o enmienda a una medida nacional relacionada con los servicios que no se ajusta a las obligaciones del acuerdo (acceso al mercado, trato nacional, trato de nación más favorecida), se realice en la dirección de una mayor conformidad con el acuerdo, no menos. Según cita el documento de la ISP esta cláusula ya ha sido aprobada.

¿Qué implicaciones tiene esta disposición para los países negociadores?, pues muy comprometedoras, porque evocan que una vez los países accedan a la liberalización de los servicios presentados, sean cuales fueran, la decisión es irreversible, en otras palabras, las medidas no podrán ser revocadas en un futuro, 
esto sin importar el costo socio económico y político para los gobiernos. Toda medida de desregulación quedara de forma permanente.

Cláusula de Status Quo. Esta disposición bloquearía los niveles actuales de liberalización de los servicios en cada país, prohibiendo con eficacia que cualquier disposición relativa a los servicios públicos basada en el mercado fuera modificada para estar basada en el Estado. Tal como cita el documento, ágilmente no prohíbe los monopolios, pero si prohíbe la creación de monopolios públicos en sectores que están actualmente abiertos a la competencia del sector privado.

Las Implicaciones de esta cláusula, en otras palabras, prohibiría que todos los servicios que sean sujetos a desregulación y privatizados, sin importar los impactos o resultados, no podrán regresar a manos de la administración del Estado, más bien continuaran abiertos a la libre oferta y competencias del mercado.

Estas dos dispersiones, de las cuales, ya la primera de Trinquete ha sido aprobada, se tornan sumamente peligrosas, toda vez que, con la filosofía conceptual neoliberal de liberalización total del mercado, sin ninguna regulación estatal se convierte en un agravante cuando dentro de los servicios están considerados los servicios públicos, los que se pretenden mercantilizar en acto de retroceso profundo sobre el sentido del derecho humano a la accesibilidad de los servicios básicos, el compromiso de los Estados de proveerlos a la población para garantizar mayor equidad y retribución, teniendo un impacto directo sobre los niveles de pobreza, que de hecho forman parte de los indicadores que se utilizan como medición de bienestar.

Incorporar también en esta negociación los servicios financieros, implicaría para los países una desprotección total a informaciones de seguridad financiera, desregular el funcionamiento de bancos y entidades financieras, dando paso a la incursión de los grandes capitales y la poca transparencia en la que se dé el establecimiento de los servicios financieros extranjeros. De igual manera el resto de los servicios como transporte, telecomunicaciones, gestión de residuos, energía, entre otros, al quedar fuera de toda regulación, expondría a sus usuarios nacionales a las especulaciones propias del mercado, sin ninguna mediad de protección.

Por último, es necesario señalar, que estas disposiciones responden de manera agresiva y fulminante a frenar las respuestas anti privatizadoras que se realizaron en algunos países de la región como Argentina después del fracaso de las políticas privatizadoras impulsadas en los años 80 y 90 . 
El TISA obliga a los gobiernos a cubrir automáticamente todos los nuevos servicios, es decir aquellos que ni siquiera existen aún, prácticamente congelando un plan de privatizaciones para las generaciones futuras (Barreto y Chávez, 2017).

De igual manera, por el investigador Ben Beachy (2020) del observatorio ciudadano sobre la globalización del comercio internacional Public Citizen Global Trade Watch, con sede en Washington, DC, cita que esta cláusula del TISA prohíbe las reglamentaciones equitativas si "inadvertidamente modifican las condiciones de competencia" en detrimento de los intereses de las grandes multinacionales, e impone a los gobiernos la obligación casi imposible de cumplir de anticipar todas las posibles regulaciones financieras -incluidas las que regularían servicios o productos todavía no inventados- que pudieran afectar de alguna manera en el futuro a las corporaciones extranjeras (Ribeiro, 1996).

En otras palabras, esta condicionante en primer lugar, obliga a los países a presentar desde el inicio de las negociaciones la lista de servicios que desean excluir de los acuerdos y todo lo que no presenten quedara bajo el dominio de los acuerdos, lo que quiere decir que en el tiempo nada quedara excluido, exige que AL menos un $90 \%$ de los servicios queden incluidos en los acuerdos y además, obliga a los países a comprometerse bajo los acuerdos no solo los servicios que se presenten al momento de iniciar las negociaciones, sino que los obliga aceptar cualquier servicio que a futuro adopten los gobiernos sin necesidad de llegar a nuevos entendimientos o firma de nuevos acuerdos en donde queden incluidos.

Los antecedentes de esta disposición, que se dieron en el seno de la OMC, habían sido rechazados y, en su lugar los Estados preferían trabajar con listas positivas. Contrario a esta propuesta, los países presentaban los servicios que querían incluir dentro de los acuerdos. Esta propuesta es peligrosa al exigir un alto porcentaje en la lista de ofertas que deben presentarse y comprometer, aun aquellos servicios que puedan surgir en el futuro, dejan al desamparo cualquier posibilidad de revertir la decisión, sujeto a las cláusulas anteriores, logrando con esto un compromiso más efectivo de liberalización y desregulación por parte del Estado sin importar los impactos de tal decisión.

Prohibición de Normas Nacionales y restricción de regulaciones locales. Esta cláusula propone que ningún servicio podrá estar sometido a ninguna norma de preferencia nacional, estableciéndose la completa apertura a la competencia internacional.

Esto implica que ya no habrá preferencia de trato nacional para ninguna empresa o servicios que brinde el Estado, si este está incluido dentro de los acuerdos, incluso cualquier ley 
o disposición nacional que ampare alguno de estos servicios, queda invalidado por los acuerdos. El impacto de esta cláusula es altamente lesivo no solo al interés primero de los Estados de proteger los intereses nacionales, sino que abre la puerta para eliminar toda restricción favorable que proteja el país y que son fundamentales para preservar su futuro. Por ejemplo, las protecciones al ambiente, al patrimonio cultural, la protección laboral a los nacionales y un sin número de servicios que quedarían afectados de manera irreversible. Incluso no podemos descartar las regulaciones para los servicios de salud, licencias farmacéuticas, educación. Recordemos que la esencia y motor que impulsan estos acuerdos es finalmente eliminar todas las barreras que hasta ahora han impedido a las multinacionales una completa desregulación del mercado para viabilizar una efectiva liberalización del comercio de servicios.

. Liberalización no diferenciada. Esta cláusula establece que los países deben liberalizar totalmente los servicios que acordó y brindar una apertura total. Esto que significa, que debe facilitar a su contraparte todas las condiciones que permitan el libre mercado, en su máxima expresión.

. Leyes no necesarias. Esta cláusula permite a cualquier multinacional o Estado miembro quejarse ante los tribunales internacionales al considerar que alguna ley o política del Estado perjudica la libre competencia.

Lo que significa que los servicios negociados y el destino económico, social y político queda a merced de los acuerdos TISA, los Estados simplemente pierden su razón de ser como entes reguladores, como garantes de políticas que promuevan equidad, bienestar a su ciudadanos y protección a los intereses nacionales. Diríamos en otras palabras, el neocolonialismo intervencionista gobernaría a nuestros países.

Divulgación. Esta cláusula es simple, las negociaciones de los acuerdos se dan a espaldas de sus ciudadanos y parlamentos, pero además obliga a sus negociadores no hacer público el contenido de resultados hasta cinco años después de haberse firmado. Su implicación directa: los países pierden su soberanía, usurpan la legitimidad del Estado, violentan derechos humanos, laborales, convenios internacionales, se pacta la entrega de servicios claves para el desarrollo económico y el bienestar de la población, pero los afectados no podrán reaccionar, puesto que ya el daño propone ser irreversible.

\section{Panamá y el TISA}

El 75\% de la economía de Panamá está basada en el sector servicios. Esto implica que nuestro país, al formar partes de las negociaciones del TISA, puede estar comprometiendo un 
porcentaje importante de los servicios que constituyen su fortaleza económica, como los son los servicios del Canal de Panamá, el centro financiero, energía, transporte, logística, por mencionar algunos.

\section{La dirección del Estado y el TISA}

En mayo del 2013 el entonces Ministro de Comercio e Industrias de Panamá, Ricardo Quijano, señalaba: “el futuro Acuerdo en Comercio de Servicios que estaría por negociarse, no sólo pretende mejorar en gran medida algunas de las disposiciones del Acuerdo General sobre el Comercio de Servicios, sino que vislumbra adicionar nuevas y mejoradas disciplinas en materias de servicios financieros, telecomunicaciones, comercio electrónico, servicios marítimos y logísticos, de alto interés para el sector privado panameño". Lo expresado pone en evidencia con una claridad impecable, que lo importante aquí es el lucro y no el interés público ni la protección de los derechos de los consumidores. Más adelante confirmaba que TISA va más allá del Acuerdo General sobre el Comercio de Servicios de la OMC, al asegurar que abarcaría "potencialmente más disciplinas y sectores no contemplados en algunos de nuestros acuerdos bilaterales".

Augusto Arosemena, el ministro de Comercio e Industrias, dejó claro ante el pleno de la Asamblea Nacional que Panamá no forma parte de los más de 50 países miembros del acuerdo de Comercio en Servicios e Inversión, mejor conocida por sus siglas en inglés como TISA.

Arosemena sustentó ante los diputados un cuestionario de seis preguntas en el cual señaló que no se ha negociado ni se pretende negociar ningún tipo de convenio o tratado comercial que afecte los más claros intereses del sector profesional del país.

Manifestó que estas negociaciones se han estado manejando con total transparencia, y se ha estado consultando con los diversos sectores involucrados y, la fecha Panamá no ha asumido ningún tipo de compromiso que permita la entrada de profesionales extranjeros.

Sobre el fundamento jurídico o la normativa legal que establece la República de Panamá como signataria de los acuerdos en comercio, servicios e inversión indicó el ministro del MICI que las profesiones liberales han quedado excluidas en todas las negociaciones comerciales que Panamá ha realizado mundialmente.

Arosemena aclaró que Panamá cuenta en estos momentos con una economía consolidada, principalmente, en el sector servicios y, por consiguiente, se tomó la decisión de incorporarse a 
la negociación de TISA, en el 2013, debido a que los participantes representan un 70 por ciento del comercio mundial de servicios.

El ministro de Comercio precisó que las negociaciones de TISA están actualmente suspendidas desde diciembre de 2016, por no alcanzar los consensos necesarios.

Esto tendría un gran impacto para la economía y el bienestar social, ya que otorgarlos a la administración de las grandes multinacionales, implicaría una descapitalización y fuga de capitales y riquezas que puede generar el país para su desarrollo económico.

Tras nacionalizar, por ejemplo, servicios públicos, servicios de transporte, permitir la participación de los grandes monopolios multinacionales afectaría directamente el bienestar de la población, puesto que encarecería aún más la vida y profundizaría y aumentaría la pobreza.

Solamente pensar que los servicios de mantenimiento del Canal sean tercerizados, implica no solo desprotección laboral, la posibilidad de aumentar el desempleo, desmejorar los salarios, sino mucho más allá, los beneficios que brinda el Canal al país que están por el orden de los 1,600 millones de dólares anuales, quedarían a merced de engrosar las arcas de los capitales corporativos, sin contar la lesión a la memoria histórica de la lucha y sangre derramada para lograr la descolonización.

Pese a las palabras del Ministro Arosemena debemos advertir que el país en los últimos años ha venido allanando el camino para la viabilidad de estos nefastos acuerdos, mediante la flexibilización laboral, la más reciente en medio de la pandemia, la promoción de leyes para ir dando pases a la instalación en el país de capitales corporativos, la más reciente también la Ley 355 EMMA, que es un régimen especial y preferencial para el establecimiento de multinacionales que prestan servicios de manufactura, so pretexto de atraer la inversión extranjera y la promoción de empleo, pero que al mismo tiempo es acompañada de sistemas preferenciales y eliminación de aranceles, además encuentra al país en una de las más terribles violaciones a los derechos humanos y laborales de los trabajadores tendientes a la reducción de salarios. Podríamos decir el ambiente perfecto y codiciado por las grandes corporaciones.

Pese a la vigilancia de las organizaciones civiles y de trabajadores, desde el año 2014, la pandemia ha sido muy bien aprovechada debido a la inmovilización de la población para dar paso a estas leyes que no descartamos seguirán aprobándose en vías de ir cumpliendo la apertura necesaria para el momento en que concluyan las negociaciones TISA.

\section{Las fuerzas sociales y el TISA}


Las organizaciones sociales panameñas llamaron al gobierno a considerar la opinión de los sectores populares, debido a "lo peligroso y pernicioso" que pueden resultar esas negociaciones para Panamá. Si no son consultados los actores sociales, laborales, políticos y económicos, está claro que se viola flagrantemente la Constitución, la ley, y se socava nuestra dignidad democrática.

No es secreto que las partes están negociando otras áreas específicas, como la regulación nacional, la transparencia y la ubicación. Son claros los aviesos propósitos de los Estados Unidos, particularmente interesado en la liberalización de los servicios financieros, tecnologías de la información y la comunicación, los servicios.

Dirigentes de organizaciones de consumidores y sindicatos panameños denunciaron que el denominado Acuerdo Internacional sobre Comercio de Servicios (TISA), representa una amenaza en la posible privatización de servicios del Canal de Panamá y empresas del Estado con una importante función social.

Pedro Acosta, secretario general de la Unión Nacional de Consumidores y Usuarios de la República de Panamá (Uncurepa), comentó durante un seminario coauspiciado por la Fundación Friedrich Ebert, de Alemania, que "las conquistas soberanas están en riesgo", a causa de la negociación del TISA, promovido por las mayores multinacionales del mundo. (Carrasco, 2019).

“Con el TISA, los servicios de agua, transporte y mantenimiento en el Canal de Panamá ampliado, son susceptibles a ser tercerizados. Por ello, hay que salir de las negociaciones del TISA", aseguró el dirigente quien promueve un frente de resistencia al acuerdo.

Anastasio Rodríguez, de la Fundación Friedrich Ebert, aseveró que el proyecto del TISA vulnera la capacidad de los Estados, al afectar derechos laborales y promover la contratación de jóvenes sin derechos laborales en procura de un crecimiento económico, pero sin la estabilidad y bienestar que exigen los trabajadores. (Carrasco, 2019).

Toribio Díaz, ex director ejecutivo de la Asociación Panameña para el Desarrollo Social de la Comunicación Social (Apadicos), manifestó que es necesario informar y advertir a la población sobre la riesgosa comercialización de los servicios públicos y la vulneración de los derechos laborales que se derivan del TISA: (Cárdenas, 2014; Carrasco, 2019). 
"Es necesario democratizar el tema entre los comunicadores nacionales" Añadió que "hay que sacar el tema de la esfera las negociaciones secretas emprendidas por Panamá", y que han provocado rechazos similares en Alemania y España, acotó.

Alfredo Berrocal, exsecretario general de la Federación Nacional de Servidores Públicos (FENASEP), indicó en su vivencia que el TISA", perjudica, a la agricultura, el empleo, la salud y los recursos hídricos de este país que ya apunta a la privatización de los servicios públicos que están bajo la responsabilidad del Estado y recursos vitales, como el agua, que podrían ser controlados por el sector privado a su manera.

\section{Sector salud y el TISA}

Busca reformar los sistemas nacionales de salud pública, las cuales serán férreas promotoras de la deslocalización de los servicios de atención de la salud y la privatización a gran escala.

Actualmente se esbozó un anexo relativo a los servicios de atención a la salud (o a la facilitación de la movilidad de los pacientes). El anexo crea un sistema basado en tres pilares que son: la financiación (reembolso), la prestación del servicio (calidad y normas) y finalmente las responsabilidades posteriores al tratamiento.

Este anexo establece que:

En el caso de la salud es evidente los postulados que encajan con los propósitos del TISA como lo refleja las reacciones de importantes sectores frente a la tercerización de servicios, mantiene en alerta a gremios y pacientes. La contratación de servicios de terceros por parte de la Caja de Seguro Social (CSS), que actualmente enfrenta una de las peores crisis que ha sufrido el sistema de salud pública del país, ha despertado el rechazo de usuarios y trabajadores, que aseguran que se trata de negocios entre la cúpula de la entidad y la empresa privada en asocio con las grandes trasnacionales de la salud. ejemplo palpable del fenómeno en discusión lo constituyó el anuncio del ex del director de la CSS, Estivenson Girón, sobre el alquiler de 97 ambulancias por cinco años a un costo de 24 millones de dólares que despertó el debate el debate sobre la forma en que se administran esos recursos del Estado.

La insatisfacción de pacientes, sindicatos y gremios médicos con la actual gestión del sistema de salud pública se ve reflejada en el creciente número de protestas, que aseguran no cesarán hasta lograr las respuestas requeridas 
La COMENENAL viene denunciando las intenciones del gobierno de mantener la externalización de los servicios públicos de salud. Esta vez los asociados se refirieron a la supuesta intención del MINSA, por intermedio de su Unidad de Gestión Administrativa y Financiera (UGAF), de renovar contratos con las Organizaciones Extrainstitucionales (OE’s) para llevar a cabo la atención de salud en las áreas apartadas del país.

De acuerdo con los expertos, la externalización de los servicios públicos de salud constituye una evidencia tangible de la incapacidad de las organizaciones públicas (en nuestro caso MINSA y CSS) para prestar los servicios que le corresponden, de forma eficiente, con equidad y calidad. Esta práctica es inadmisible en un país con los recursos suficientes como el nuestro. No obstante, se ha convertido en un ejercicio común en la gestión pública de los últimos gobiernos (por ejemplo: reglamento de prestaciones, cirugías cardiacas, hemodiálisis, etc.), los que han esgrimido las más variadas explicaciones para contratar en el sector privado prestaciones y procedimientos que bien podría brindar un sector público fortalecido

El TISA en Panamá busca la privatización de los servicios salud del país, mediante la unificación de la CSS y el MINSA, donde este último será el órgano rector y planificador de la administración pública de salud, ejerciendo la competencia privativa en todo lo relacionado con las adquisiciones de insumos, instrumental, equipos y otros productos que requieran todas las instituciones públicas de salud.

Encaminado a profundizar la tendencia de socavar los servicios públicos de salud y convertirlos en un sistema regulado por el mercado los ciudadanos serán clientes de un mercado globalizado de la salud.

Como lo destaca el Dr. Fernando Cebamanos: 'Con esta modalidad el sistema público contará con menos fondos, invertirá menos en instalaciones, personal y equipos. Los fondos de los contribuyentes se transfieren a la atención privada en otro país. Los servicios de salud locales funcionarán como exportadores de salud y serán 'acreditados' para la atención local, que terminará en centros privados, aumentando la desigualdad en la atención de la salud'. (López, 2015). Así se comenzaría la comercialización de la salud donde solo unos pocos se beneficiarían, muchas estructuras pasaran a manos privadas, tras nacionalizando los servicios de la salud.

\section{Sector Educativo}

La definición, parámetros en cuanto al sistema educativo no precisa interpretaciones, especificas, sin embargo, los procesos de privatización son evidente mediante el abandono de la calidad e infraestructura de los centros de educación pública, y las concesiones a las empresas 
vinculada a la educación nuevos derechos, restringiría la capacidad de regular la actividad de los proveedores y finalmente esto limitaría al gobierno de reglamentar y proporcionar una educación eficaz y de calidad.

En cuanto a la Educación a Nivel Superior es evidente la política hacia una profundización de los procesos de privatización, minimizando la dotación de los recursos para el cumplimiento de los servicios esenciales de las universidades públicas, contrario a las concesiones y privilegios a los inversionistas privados y extranjeros dedicados a el negocio de la educación.

Lo que es a juicio del lingüista Noam Chomsky: "El neoliberalismo intenta tomar por asalto las universidades" bajo el principio o modelo empresarial, pretendiendo inscribir las instituciones de educación superior en un estado de precarización de la calidad de la enseñanza y el aprendizaje.” (Luna Mendoza, 2014).

Enmarcados en los propósitos y principios de la transnacionalización de la Educación a Nivel Superior la Asociación de Universidades Particulares de Panamá (AUPPA), en conjunto con representantes del Órgano Legislativo, perteneciente a la Comisión de Gobierno, propiciaron la modificación de los artículos 99 y 104 del numeral 2 del artículo 313 título XIII de la Constitución Política.

"Respaldamos la iniciativa de revisión del artículo que en cuanto a la educación superior se refieren, por ser cambios que nuestra sociedad pide a gritos de acuerdo a las mediciones realizadas en desempeños de la competitividad de nuestro país y la necesidad de capital humano actualizado frente a un mundo cambiante minuto a minuto", señala AUPPA.

Como contra propuesta la Universidad de Panamá emitió su visión entorno a los propósitos de (AUPPA) exteriorizando hay dos puntos que no compartimos con la renovación constitucional. El primero: la creación del Sistema Nacional de Evaluación y Acreditación para el Mejoramiento de la Calidad de Educación Superior de Panamá, ya que les quita a las universidades oficiales la potestad de ser la fiscalizadora de la educación superior en el país. Como segundo argumento, la Universidad de Panamá expresó que la propuesta sobre la excepción de pago de impuestos y donaciones que se otorguen a favor de las universidades, entidades culturales o científicas de carácter privada.

Las implicaciones de carácter político concebida por los promotores del modelo económico de control de los servicios públicos y bienes materiales de alto valor alcanzan nuevos estadios con la propuesta mancomunada de la Cámara de Comercio, Industrias y Agricultura de 
Panamá (CCIAP) y la Asociación Panameña de Ejecutivos de Empresas (APEDE), de atraer talento extranjero al país y eliminar restricciones en carreras protegidas para los nacionales.

"La posición del sector privado es que tenemos que ver cuáles son los beneficios concretos que trae una liberalización de esa política de migración. Que hay que revisar esa política de migración, “estudiosos en la materia han señalado la importancia que Panamá se abra a la inversión extranjera, al talento extranjero."

En contraposición al enfoque de apertura del mercado y la liberación de las medidas de restricción salvaguarda de los profesionales nacionales del capital La Sociedad Panameña de Ingenieros y Arquitectos (SPIA) señaló que "de manera reiterada, han hecho énfasis en la necesidad de cumplir con lo relacionado al ejercicio legal de la profesión que incluye la obligatoriedad de la idoneidad profesional. La posición de la CCIAP está en contraposición a los artículos 40 y 73 de la Constitución Política de la República de Panamá y la Ley 15 de 26 de enero de 1959, atentando directamente contra los profesionales de la ingeniería y arquitectura idóneos en el país".

La Asociación de Profesores de la Universidad de Panamá, rechazó lo esgrimido por la CCIAP, la cual transita por avenidas distinta al fortalecimiento de un proyecto nacional".

La Comisión Médica Negociadora Nacional (COMENENAL) rechazó la propuesta de contratar mano de obra extranjera para profesiones como arquitectos, médicos, abogados e ingenieros. Los médicos aseguran que "Panamá tiene suficientes profesionales calificados. demostrado en las diversas profesiones liberales".

En medio de la pandemia la Asociación de Universidades Particulares de Panamá (AUPPA) con la rúbrica de Adriana Angarita y José Alberto Nieto Rojas presentan a la Comisión Técnica de Desarrollo Académico la petición especial, de la suspensión de los cobros o tarifas por aprobación de planes y programas de estudios, debido a la actual situación por la cual atravesamos las universidades particulares, pues no somos ajenas a la afectación económica que vive el país, por cuanto se han visto disminuidos los ingresos por conceptos de matrícula y mensualidades".

El 18 de mayo del 2020 la Secretaria Ejecutiva del Consejo de Evaluación y Acreditación Universitaria de Panamá, Doctora María del Carmen de Benavides eleva al licenciado Virgilio Sousa, Director Nacional de Asesoría Legal del Ministerio de Educación, opinión legal sobre este tema de exclusiva responsabilidad de la Universidad de Panamá. 
El enunciado del CTDA a todas luces guardan concordancia con la letra y espíritu de los organismos internacionales de estrangulamiento, desnaturalización, desarticulación, de las Instituciones de Educación Superior de carácter público.

\section{CONSIDERACIONES FINALES.}

La reducción a su mínima expresión de la intervención del Estado en las decisiones de corte social y económica, propósito central de la estrategia económica de los sectores que detentan el poder político y económico.

Desregular la economía y las funciones de las empresas estatales "Garantes de los Servicios básicos", concediéndole mayores privilegios a los intereses transnacionales y a los fines hegemónicos consagradas en las denominadas cláusula "trinquete" y "statu quo", en las cuales los Estados son sometidos de manera indefinida a aplicar las recetas de los organismos internacionales que controlan el comercio mundial.

El libreto del TISA conduce a la pérdida de soberanía de los Estados.

\section{REFERENCIAS BIBLIOGRÁFICAS}

Abreu, S. (2015). Reflexiones sobre el TISA. Academia Nacional de Economía. Uruguay.

Assange, J. (2011). Julian Assange: The unauthorised autobiography. Edinburgh: Canongate Books.

Barreto, V. y Chávez, D. (2017). El TISA y las empresas del Estado. El Transnational Institute (TNI) y REDES Amigos de la Tierra. Montevideo, Uruguay.

Beachy, B. (2020) Análisis del texto del TISA sobre Servicios Financieros. Recuperado de: https://studylib.es/doc/2667032/an\%C3\%A1lisis-del-texto-del-tisa-sobre-serviciosfinancieros.pdf. Fecha: 23 de nov. de 20. 
Cárdenas, J. Manuel. (2014). "El TISA y los servicios públicos”. Portafolio, Revista digital. Bogotá, Colombia. 11 de mayo de 2014.

Carrasco, D. (2019) Acuerdo TISA amenaza al Canal de Panamá. Recuperado de: https://www.world-psi.org/es/acuerdo-tisa-amenaza-al-canal-de-panama. Fecha: 5 de septiembre de 2019.

Espinosa R, J. (2016). "Los acuerdos TISA: el nuevo orden mundial”. La Estrella de Panamá. Rep. de Panamá, 25 de agosto de 2016.

Klein, N. (2011). La Doctrina del Shock: el Auge del Capitalismo. Buenos Aires, Paidós Editorial.

Lange, C. (2010). La Doctrina del Shock. El auge del capitalismo del desastre. Revista INVI.

López, G. (2015). Cabalga de nuevo la "ley de la muerte" Disponible en: https://www.laestrella.com.pa/opinion/columnistas/150913/ley-nuevo-muerte-cabalga. Fecha: 08 de agosto de 2019.

Luna Mendoza, M. (2014). "El neoliberalismo tomó por asalto a las universidades": Noam Chomsky. Disponible en: https://www.elespectador.com/noticias/educacion/elneoliberalismo-tomo-por-asalto-a-las-universidades-noam-chomsky/ Fecha: 23 de otubre de 2019.

Ribeiro L. G. (1996). Globalización y transnacionalización Perspectivas antropológicas y latinoamericanas. Universidad Nacional de Colombia. Maguaré, Brasil.

Valdés, Y. (2017). “Advierten que el TISA busca vender Panamá”. Panamá América. Rep. de Panamá, 15 de agosto de 2017. 